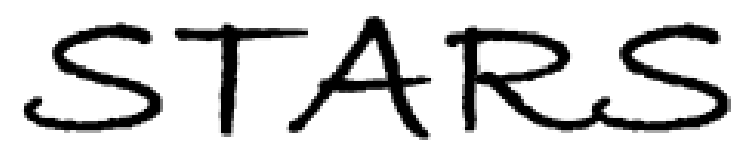

University of Central Florida

STARS

$1-1-2011$

\title{
Broadband circularly-polarized infrared emission from multilayer metamaterials
}

Samuel L. Wadsworth

University of Central Florida

Paul G. Clem

Eric D. Branson

Glenn D. Boreman

University of Central Florida

Find similar works at: https://stars.library.ucf.edu/facultybib2010

University of Central Florida Libraries http://library.ucf.edu

This Article is brought to you for free and open access by the Faculty Bibliography at STARS. It has been accepted for inclusion in Faculty Bibliography 2010 s by an authorized administrator of STARS. For more information, please contact STARS@ucf.edu.

\section{Recommended Citation}

Wadsworth, Samuel L.; Clem, Paul G.; Branson, Eric D.; and Boreman, Glenn D., "Broadband circularlypolarized infrared emission from multilayer metamaterials" (2011). Faculty Bibliography 2010s. 2054. https://stars.library.ucf.edu/facultybib2010/2054

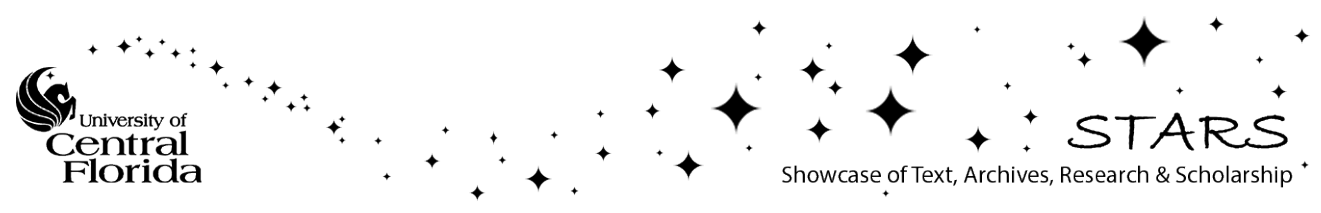




\title{
Broadband circularly-polarized infrared emission from multilayer metamaterials
}

\author{
Samuel L. Wadsworth, ${ }^{1, *}$ Paul G. Clem, ${ }^{2}$ Eric D. Branson, ${ }^{2}$ and Glenn D. Boreman ${ }^{1}$ \\ ${ }^{I}$ University of Central Florida, CREOL - The College of Optics and Photonics, 4000 Central Florida Blvd, Orlando, \\ Florida 32816, USA. \\ ${ }^{2}$ Sandia National Laboratories, Advanced Materials Laboratory, 1001 University Blvd. SE, Albuquerque, New \\ Mexico 87106 USA \\ *swadswor@creol.ucf.edu
}

\begin{abstract}
Development of a 2D metamaterial that preferentially emits broadband circularly-polarized (CP) infrared radiation is hindered by the fact that orthogonal electric-field components are uncorrelated at the surface of the thermal emitter, a consequence of the fluctuation-dissipation theorem. We achieve broadband CP thermal emission by fabricating a meanderline quarter-wave retarder on a transparent thermal-isolation layer. Behind this isolation layer, in thermal contact with the emitter, is a wire-grid polarizer. Along with an unavoidable linear polarized radiation characteristic from the meanderline, we measured a degree of circular polarization (DOCP) of $28 \%$, averaged over the 8 - to $12 \mu \mathrm{m}$ band.

(O2011 Optical Society of America

OCIS codes: (160.3918) Metamaterials; (260.5430) Polarization; (030.1640) Coherence; (030.5620) Radiative transfer; (120.5410) Polarimetry; (350.5610) Radiation.
\end{abstract}

\section{References and links}

1. F. Marquier, C. Arnold, M. Laroche, J. J. Greffet, and Y. Chen, "Degree of polarization of thermal light emitted by gratings supporting surface waves," Opt. Express 16(8), 5305-5313 (2008).

2. J. Le Gall, M. Olivier, and J.-J. Greffet, "Experimental and theoretical study of reflection and coherent thermal emissionby a SiC grating supporting a surface-phonon polariton," Phys. Rev. B 55(15), 10105-10114 (1997).

3. K. Joulain, J.-P. Mulet, F. Marquier, R. Carminati, and J.-J. Greffet, "Surface electromagnetic waves thermally excited: Radiative transfer, coherence properties and Casimir forces revisited in the near field," Surf. Sci. Rep. 57(3-4), 59-112 (2005).

4. P. J. Hesketh, J. N. Zemel, and B. Gebhart, "Polarized spectral emittance from periodic micromachined surfaces. II. Doped silicon: Angular variation,” Phys. Rev. B Condens. Matter 37(18), 10803-10813 (1988).

5. M. Laroche, C. Arnold, F. Marquier, R. Carminati, J.-J. Greffet, S. Collin, N. Bardou, and J.-L. Pelouard, "Highly directional radiation generated by a tungsten thermal source," Opt. Lett. 30(19), 2623-2625 (2005).

6. V. Demir, I. E. Araci, A. Kropachev, T. Skotheim, R. A. Norwood, and N. Peyghambarian, "Nanoamorphous carbon as a blackbody source in plasmonic thermal emitters," Appl. Opt. 50(2), 218-221 (2011).

7. Y. Ueba, J. Takahara, and T. Nagatsuma, "Thermal radiation control in the terahertz region using the spoof surface plasmon mode," Opt. Lett. 36(6), 909-911 (2011).

8. N. Dahan, A. Niv, G. Biener, Y. Gorodetski, V. Kleiner, and E. Hasman, "Extraordinary coherent thermal emission from SiC due to coupled resonant cavities," J. Heat Transfer 130(11), 112401 (2008).

9. T. Setälä, M. Kaivola, and A. T. Friberg, "Degree of polarization in near fields of thermal sources: effects of surface waves," Phys. Rev. Lett. 88(12), 123902 (2002).

10. A. V. Shchegrov, K. Joulain, R. Carminati, and J.-J. Greffet, "Near-field spectral effects due to electromagnetic surface excitations," Phys. Rev. Lett. 85(7), 1548-1551 (2000).

11. M. Laroche, R. Carminati, and J.-J. Greffet, "Coherent thermal antenna using a photonic crystal slab," Phys. Rev. Lett. 96(12), 123903 (2006).

12. B. J. Lee and Z. M. Zhang, "Coherent thermal emission from modified periodic multilayer structures," J. Heat Transfer 129(1), 17-26 (2007).

13. M. Florescu, H. Lee, A. J. Stimpson, and J. Dowling, "Thermal emission and absorption of radiation in finite inverted-opal photonic crystals," Phys. Rev. A 72(3), 033821 (2005).

14. S. Enoch, J.-J. Simon, L. Escoubas, Z. Elalmy, F. Lemarquis, P. Torchio, and G. Albrand, "Simple layer-by-layer photonic crystal for the control of thermal emission,” Appl. Phys. Lett. 86(26), 261101 (2005).

15. M. Garín, T. Trifonov, D. Hernández, A. Rodriguez, and R. Alcubilla, "Thermal emission of macroporous silicon chirped photonic crystals," Opt. Lett. 35(20), 3348-3350 (2010).

16. D. L. C. Chan, M. Soljacić, and J. D. Joannopoulos, "Thermal emission and design in one-dimensional periodic metallic photonic crystal slabs,” Phys. Rev. E Stat. Nonlin. Soft Matter Phys. 74(1), 016609 (2006). 
17. S.-Y. Lin, J. G. Fleming, E. Chow, J. Bur, K. K. Choi, and A. Goldberg, "Enhancement and suppression of thermal emission by a three-dimensional photonic crystal," Phys. Rev. B 62(4), R2243-R2246 (2000).

18. N. Dahan, A. Niv, G. Biener, V. Kleiner, and E. Hasman, "Space-variant polarization manipulation of a thermal emission by a $\mathrm{SiO}_{2}$ subwavelength grating supporting surface phonon-polaritons," Appl. Phys. Lett. 86(19), $191102(2005)$.

19. J.-H. Lee, J. C. W. Lee, W. Leung, M. Li, K. Constant, C. T. Chan, and K.-M. Ho, "Polarization engineering of thermal radiation using metallic photonic crystals," Adv. Mater. (Deerfield Beach Fla.) 20(17), 3244-3247 (2008).

20. J. C. W. Lee and C. T. Chan, "Circularly polarized thermal radiation from layer-by-layer photonic crystal structures," Appl. Phys. Lett. 90(5), 051912 (2007).

21. O. G. Kollyukh, A. I. Liptuga, V. Morozhenko, V. I. Pipa, and E. F. Venger, "Circular polarized coherent thermal radiation from semiconductor layers in an external magnetic field," Opt. Commun. 276(1), 131-134 (2007).

22. G. P. Nordin, J. T. Meier, P. C. Deguzman, and M. W. Jones, "Micropolarizer array for infrared imaging polarimetry," J. Opt. Soc. Am. A 16(5), 1168-1174 (1999).

23. M. W. Kudenov, J. L. Pezzaniti, and G. R. Gerhart, "Microbolometer-infrared imaging Stokes polarimeter,” Opt. Eng. 48(6), 063201 (2009).

24. F. Gori, "Measuring Stokes parameters by means of a polarization grating," Opt. Lett. 24(9), 584-586 (1999).

25. J. Ginn, D. Shelton, P. Krenz, B. Lail, and G. Boreman, "Polarized infrared emission using frequency selective surfaces," Opt. Express 18(5), 4557-4563 (2010).

26. D. Goldstein, Polarized Light, (Marcel Dekker, 2003).

27. E. C. Zimmermann and A. Dalcher, "Incoherent radiative properties of an opaque body," J. Opt. Soc. Am. A 8(12), 1947-1954 (1991).

28. L. Mandel and E. Wolf, Optical Coherence and Quantum Optics, (Cambridge, 1995).

29. L. Novotny and B. Hecht, Principles of Nano-Optics, (Cambridge, 2006).

30. S. M. Rytov, Y. A. Kravtsov, and V. I. Tatarskii, Principles of Statistical Radiophysics 3: Elements of Random Fields, (Springer-Verlag, 1989).

31. L. D. Landau and E. M. Lifshitz, Electrodynamics of Continuous Media, (Pergamon Press, 1960).

32. G. S. Agarwal, "Quantum electrodynamics in the presence of dielectrics and conductors. I. Electromagnetic-field response functions and black-body fluctuations in finite geometries," Phys. Rev. A 11(1), 230-242 (1975).

33. C. Henkel, K. Joulain, R. Carminati, and J.-J. Greffet, "Spatial coherence of thermal near fields," Opt. Commun. 186(1-3), 57-67 (2000).

34. N. R. Labadie and S. K. Sharma, "A novel compact volumetric metamaterial structure with asymmetric transmission and polarization conversion," Metamaterials (Amst.) 4(1), 44-57 (2010).

35. A. Resnick, C. Persons, and G. Lindquist, "Polarized emissivity and Kirchhoff's law," Appl. Opt. 38(8), 13841387 (1999).

36. J. J. Greffet and M. Nieto-Vesperinas, "Field theory for generalized bidirectional reflectivity: derivation of Helmholtz's reciprocity principle and Kirchhoff's law," J. Opt. Soc. Am. A 15(10), 2735-2744 (1998).

37. L. Tsang, J. A. Kong, and K. H. Ding, Scattering of Electromagnetic Waves. Theories and Applications, (J. Wiley, 2000).

38. J. S. Tharp, J. M. Lopez-Alonso, J. C. Ginn, C. F. Middleton, B. A. Lail, B. A. Munk, and G. D. Boreman, "Demonstration of a single-layer meanderline phase retarder at infrared," Opt. Lett. 31(18), 2687-2689 (2006).

39. S. L. Wadsworth and G. D. Boreman, "Analysis of throughput for multilayer infrared meanderline waveplates," Opt. Express 18(13), 13345-13360 (2010).

40. M. Schmidt and F. Schwertfeger, “Applications for silica aerogel products,” J. Non-Cryst. Solids 225, 364-368 (1998).

41. L. W. Hrubesh and R. W. Pekala, "Thermal properties of organic and inorganic aerogels," J. Mater. Res. 9(3), 731-738 (1994).

42. J. A. Ruffner, P. G. Clem, B. A. Tuttle, C. J. Brinker, C. S. Sriram, and J. A. Bullington, "Uncooled thin film infrared imaging device with aerogel thermal isolation: deposition and planarization techniques," Thin Solid Films 332(1-2), 356-361 (1998).

43. P. E. Hopkins, B. Kaehr, L. M. Phinney, T. P. Koehler, A. M. Grillet, D. Dunphy, F. Garcia, and C. J. Brinker, "Measuring the thermal conductivity of porous, transparent $\mathrm{SiO} 2$ films with time domain thermoreflectance," J. Heat Transfer 133(6), 061601 (2011).

44. P. E. Hopkins, B. Kaehr, E. S. Piekos, D. Dunphy, and C. J. Brinker, "Minimum thermal conductivity considerations in aerogel thin films," manuscript in preparation (2011).

45. W. R. Folks, J. C. Ginn, D. J. Shelton, J. S. Tharp, and G. D. Boreman, "Spectroscopic ellipsometry of materials for infrared micro-device fabrication," Phys. Status Solidi 5(5), 1113-1116 (2008) (c).

\section{Introduction}

There have recently been an increasing number of studies related to the thermal emission properties of microstructured surfaces and multilayered photonic structures [1-18]. By tailoring the local optical density of states via excitation of coherent surface waves $[2,3,8-$ 10,18], microstructured surfaces can exhibit thermal properties not encountered in nature, such as coherent narrow-band thermal emission and highly-directional far-field radiation by 
grating momentum transfer coupled to a surface phonon polariton [1-8,18]. Likewise, multilayered photonic crystal structures have shown to exhibit control of thermal emissive properties, when multiple Bragg scattering yields highly-directional, narrow-band thermal radiation [11-14] with significant control over the spectral emissivity [15-17]. These grating structures have been demonstrated to produce highly polarized radiation $[1-4,18]$. Multilayer photonic crystals have also been investigated as discrete polarization-state emitters [19], with one such study proposing a method to produce broadband circularly polarized $(\mathrm{CP})$ emission via a complete polarization band gap [20]. Experimentally, broadband CP thermal emission has only been demonstrated to date using thermal excitation of a uniaxial anisotropic material, which is anisotropic because it is placed in a magnetic field [21].

Because thermal infrared (IR) radiation from the natural environment is not $\mathrm{CP}$, there are applications where it is desirable to produce broadband CP radiation. This could find utility in imaging polarimetry, allowing for selection of objects based upon their emitted state of polarization [22-24]. In particular, a low-profile conformal structure is desired that has a distinct CP broadband thermal emission characteristic. It has already been demonstrated that a planar frequency-selective surface (FSS) comprised of lossy dipole elements produces a linearly polarized (LP) field when thermally excited [25]. In this paper, we investigate ways to produce an analogous IR FSS structure for broadband CP radiation.

\section{Coherence of cross-polarized thermally-emitted field components}

While LP radiation can be produced by the dipole FSS arrangement of [25], configuration of a structure with preferential radiation for $\mathrm{CP}$ is much more difficult because transverse coherence between two components of the electromagnetic field is required. Since thermal radiation is unpolarized [26-28], this characteristic must be considered in the design of any potential CP FSS. The coherence properties of thermally emitted fields in the infrared (IR) spectrum are given by the Wolf coherence matrix of a time-dependent electromagnetic field

$$
W(x, y, t)=\left[\begin{array}{ll}
\left\langle E_{s}(x, y, t)^{*} \cdot E_{s}(x, y, t)\right\rangle & \left\langle E_{s}(x, y, t)^{*} \cdot E_{p}(x, y, t)\right\rangle \\
\left\langle E_{p}(x, y, t)^{*} \cdot E_{s}(x, y, t)\right\rangle & \left\langle E_{p}(x, y, t)^{*} \cdot E_{p}(x, y, t)\right\rangle
\end{array}\right],
$$

where the field terms $E_{s, p}$ denote the $s$ and $p$-polarized components of the electric-field vector in the plane of the polarization ellipse, '*' denotes complex conjugation, and the brackets $<>$ denote the ensemble average of the analytic signal taken over a successive series of measurements or time intervals [26,28]. Equation (1) is representative of the timedependent irradiance that would be detected by broadband imaging methods, since the wavelength dependence is averaged for a broadband imager. Thus, Eq. (1) accounts for the information that would be available from images: spatial dependence of the time-averaged irradiance.

Evaluation of the matrix involves the co-polarized and cross-polarized irradiance, since each matrix element is an inner product between amplitudes of the same or orthogonal polarization components. With respect to polarized emissivity, the field amplitude can be taken as proportional the square root of the emissivity corresponding to either the $s$ - or $p$ polarized state. Since the field amplitudes are inherently complex, the square root of the polarized emissivity would also be complex in value as well, so that Eq. (1) would be written as

$$
W(x, y, t)=\left[\begin{array}{ll}
\left\langle{\sqrt{\varepsilon_{s}(x, y, t)}}^{*} \cdot \sqrt{\varepsilon_{s}(x, y, t)}\right\rangle & \left\langle{\sqrt{\varepsilon_{s}(x, y, t)}}^{*} \cdot \sqrt{\varepsilon_{p}(x, y, t)}\right\rangle \\
\left\langle{\sqrt{\varepsilon_{p}(x, y, t)}}^{*} \cdot \sqrt{\varepsilon_{s}(x, y, t)}\right\rangle & \left\langle\sqrt{\varepsilon_{p}(x, y, t)} \cdot \sqrt{\varepsilon_{p}(x, y, t)}\right\rangle
\end{array}\right],
$$

where $\varepsilon_{s, p}$ are the $s$ and $p$-polarized components of the emissivity. Now that we have defined the coherence matrix in terms of polarized emissivities, the Stokes vector of the polarization

$\# 148230$ - \$15.00 USD

(C) 2011 OSA 
signal can be formulated from the individual elements of the coherence matrix of Eq. $[1,26,28]$.

$$
\bar{S}(x, y, t)=\left[\begin{array}{c}
S_{0} \\
S_{1} \\
S_{2} \\
S_{3}
\end{array}\right]=\left[\begin{array}{c}
\left\langle{\sqrt{\varepsilon_{s}(x, y, t)}}^{*} \cdot \sqrt{\varepsilon_{s}(x, y, t)}\right\rangle+\left\langle{\sqrt{\varepsilon_{p}(x, y, t)}}^{*} \cdot \sqrt{\varepsilon_{p}(x, y, t)}\right\rangle \\
\left\langle{\sqrt{\varepsilon_{s}(x, y, t)}}^{*} \cdot \sqrt{\varepsilon_{s}(x, y, t)}\right\rangle-\left\langle\sqrt{\varepsilon_{p}(x, y, t)} \cdot \sqrt{\varepsilon_{p}(x, y, t)}\right\rangle \\
\left\langle{\sqrt{\varepsilon_{s}(x, y, t)}}^{*} \cdot \sqrt{\varepsilon_{p}(x, y, t)}\right\rangle+\left\langle\sqrt{\varepsilon_{p}(x, y, t)} \cdot \sqrt{\varepsilon_{s}(x, y, t)}\right\rangle \\
i \cdot\left(\left\langle{\sqrt{\varepsilon_{p}(x, y, t)}}^{*} \cdot \sqrt{\varepsilon_{s}(x, y, t)}\right\rangle-\left\langle\sqrt{\varepsilon_{s}(x, y, t)} \cdot \sqrt{\varepsilon_{p}(x, y, t)}\right\rangle\right.
\end{array}\right],
$$

where the above formula can be re-written as (the dependence upon $(\mathrm{x}, \mathrm{y}, \mathrm{t})$ is assumed)

$$
\bar{S}=\left[\begin{array}{c}
S_{0} \\
S_{1} \\
S_{2} \\
S_{3}
\end{array}\right]=\left[\begin{array}{c}
\left\langle\varepsilon_{s}\right\rangle+\left\langle\varepsilon_{p}\right\rangle \\
\left\langle\varepsilon_{s}\right\rangle-\left\langle\varepsilon_{p}\right\rangle \\
2 \cdot\left\langle\sqrt{\varepsilon_{s} \cdot \varepsilon_{p}} \cdot \cos (\delta)\right\rangle \\
2 \cdot\left\langle\sqrt{\varepsilon_{s} \cdot \varepsilon_{p}} \cdot \sin (\delta)\right\rangle
\end{array}\right]=\left[\begin{array}{c}
\left\langle\varepsilon_{s}\right\rangle+\left\langle\varepsilon_{p}\right\rangle \\
\left\langle\varepsilon_{s}\right\rangle-\left\langle\varepsilon_{p}\right\rangle \\
\left\langle\varepsilon_{+45^{\circ}}\right\rangle-\left\langle\varepsilon_{-45^{\circ}}\right\rangle \\
\left\langle\varepsilon_{R H C P}\right\rangle-\left\langle\varepsilon_{L H C P}\right\rangle
\end{array}\right],
$$

where the variable $\delta$ is the phase shift between the two orthogonal components of the polarized emissivity. The Stokes vector gives a direct physical interpretation of the state of polarization of a given electromagnetic field. In each element of the Stokes vector, the irradiance associated with a particular state of polarization is quantified by the measured difference between orthogonal polarization states [26,28]. Since we are working with polarized emissivity, we can express the Stokes vector components in terms of the emissivity corresponding to each measured polarization state, as shown in Eq. (4).

We now consider the cross-polarization coherence that must exist to yield a non-zero correlation between the cross-polarized components [3,26,28]. From Eqs. (2) and (3), one can readily discern the need for correlated cross-polarized components in order to obtain $\pm 45^{\circ} \mathrm{LP}$ or right-handed/left-handed circularly polarized (RHCP/LHCP) radiation. Both off-diagonal components of Eq. (2) appear in the expressions for $S_{2}$ and $S_{3}$. This correlation between crosspolarized components is specified by the normalized coherence function between crosspolarized states:

$$
j_{s p}=\frac{\left\langle\sqrt{\varepsilon_{s}(x, y, t)} \cdot \sqrt{\varepsilon_{p}(x, y, t)}\right\rangle}{\left\langle{\sqrt{\varepsilon_{s}(x, y, t)}}^{*} \cdot{\sqrt{\varepsilon_{s}(x, y, t)}}^{*}\right\rangle^{1 / 2} \cdot\left\langle{\sqrt{\varepsilon_{p}(x, y, t)}}^{*} \cdot \sqrt{\varepsilon_{p}(x, y, t)}\right\rangle^{1 / 2}},
$$

In order to obtain a finite component of $\mathrm{CP}$ in the polarization spectrum of the thermally emitted field, the value of Eq. (5) must be non-zero and imaginary. Thus, the correlation of cross-polarized field components is absolutely necessary for CP emission.

Radiative emission from a structure that is thermally excited can be thought of as a stochastic process by which the current density on the surface of the structure takes on random magnitudes, phases, and directions at each instance of excitation [29-31]. Thus, to portray the electromagnetic fields that arise from thermal excitation, one has to consider the fluctuational electrodynamics of the problem. In the case of polarized thermal emission from a planar periodic FSS, the elements of the coherence matrix of Eq. (2) can be fully determined from the Fluctuation-Dissipation Theorem (FDT) [3,9,10,29-33]. The FDT was used to analytically verify the extreme narrowband coherent emission behavior of surface-relief gratings that support p-polarized surface-wave modes [1-8]. However, the implications of the FDT are not

$\# 148230$ - \$15.00 USD

(C) 2011 OSA 
favorable for broadband $\mathrm{CP}$ emission. First of all, the FDT negates any cross-correlation of field components, even over finite-dimension geometries [31,32] of the FSS unit cell. This is equivalent to saying that the normalized coherence function of Eq. (5) will always result in a value of 0 , since orthogonal current modes from random thermal excitations will be completely uncorrelated in phase [29-33]. Secondly, there is no intrinsic anisotropy in the planar FSS that would allow for CP emission. Since the dimensions associated with the thermal-excitation process are within the electron-scattering length, the associated anisotropy would be required to exist within that same length scale, which is approximately the Fermi electron wavelength of the radiating material [33]. Thus, the FDT results in zero correlation between orthogonal current and field modes, even for finite subwavelength structures. Finally, for any structure that emits broadband radiation, the transverse coherence length becomes inherently smaller as the span of operational frequencies becomes larger $[2,3,29,33]$. This is a consequence of the Wiener-Khinchin theorem and Fourier transform laws of the mutual coherence function of a thermal electromagnetic field. As one expands the spectral domain considered, the respective correlation length must decrease $[2,28,29]$. Thus, from a theoretical standpoint it appears that CP thermal emission from a purely planar FSS structure is not allowed. However, we will design a multilayer FSS that is able to avoid these constraints and provide a significant amount of broadband CP radiation in the IR.

\section{FSS designs for investigating broadband CP emission}

Considering the fundamental limitations imposed by the FDT on the functionality of thermally emissive CP FSS arrays, our goal for the investigation of FSS designs is two-fold: 1) experimentally demonstrate the implications of the FDT regarding $\mathrm{CP}$ emission, and 2) design a multilayer FSS that avoids the constraints of the FDT, and emits significant broadband $\mathrm{CP}$ radiation. We choose the wavelength range of interest as the long-wave infrared (LWIR) $8-12 \mu \mathrm{m}$ band.

The first set of devices was designed in the ANSYS High Frequency Structure Simulator (HFSS), a full-wave finite-element solver that models FSS structures through the use of Floquet ports [34]. We investigated two planar FSS designs that were simulated and fabricated to demonstrate the implications of the FDT. Figures 1 a) and b) illustrate the unit cells for both FSS designs. Each FSS was comprised of Titanium (Ti) elements modeled on top of a $1.2-\mu \mathrm{m}$ thick layer of Benzocyclobutene (BCB), on a reflective Aluminum (Al) groundplane (Fig. $1 \mathrm{c}$ ).

\#148230 - \$15.00 USD

(C) 2011 OSA 

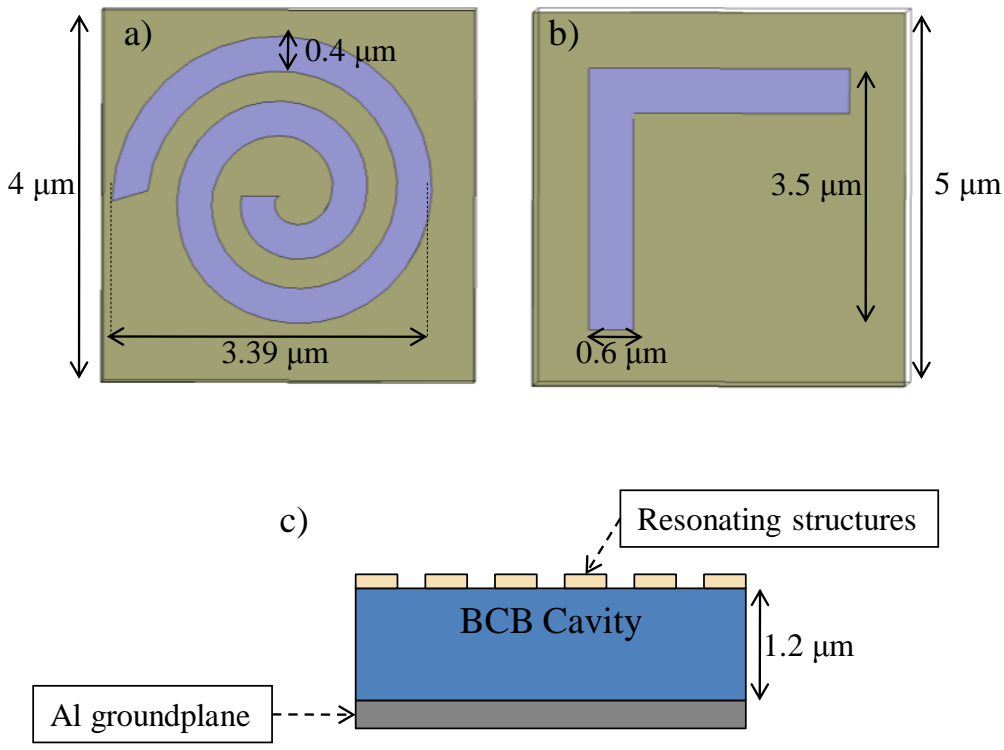

Fig. 1. Unit cell profiles of a) circular spiral array, and b) L-shaped wedge array. Below c) is a cross-sectional profile of the emissive structures as simulated in HFSS.

The polarized emissivity from both designs was computed by calculating the power reflection coefficient at normal incidence, using Kirchhoff's law to determine the emissivity corresponding to each polarization state [30,35-37]. The spectral emissivity for a purely reflective device is given as

$$
\varepsilon(\lambda, \mathrm{T})=A(\lambda, \mathrm{T})=1-R(\lambda, \mathrm{T}),
$$

where $\lambda$ the wavelength of radiation, $A$ and $R$ are the absorptivity and reflectivity of the composite metamaterial, and $\mathrm{T}$ is the temperature in Kelvin of the emissive structure. To obtain the polarization components of the Stokes vector, given by Eq. (4), the polarized emissivity coefficients are determined as

$$
\begin{gathered}
\varepsilon_{s, p}=1-R_{s, p}, \\
\varepsilon_{ \pm 45^{\circ}}=1-R_{ \pm 45^{\circ}}, \\
\varepsilon_{R H C P, L H C P}=1-R_{R H C P, L H C P} .
\end{gathered}
$$

Once these are obtained, the Stokes vector components are readily available via direct insertion of the above expressions into Eq. (4). Figure 2 illustrates the simulated Stokes parameters that were calculated by HFSS for the planar FSS designs shown in Fig. 1. 

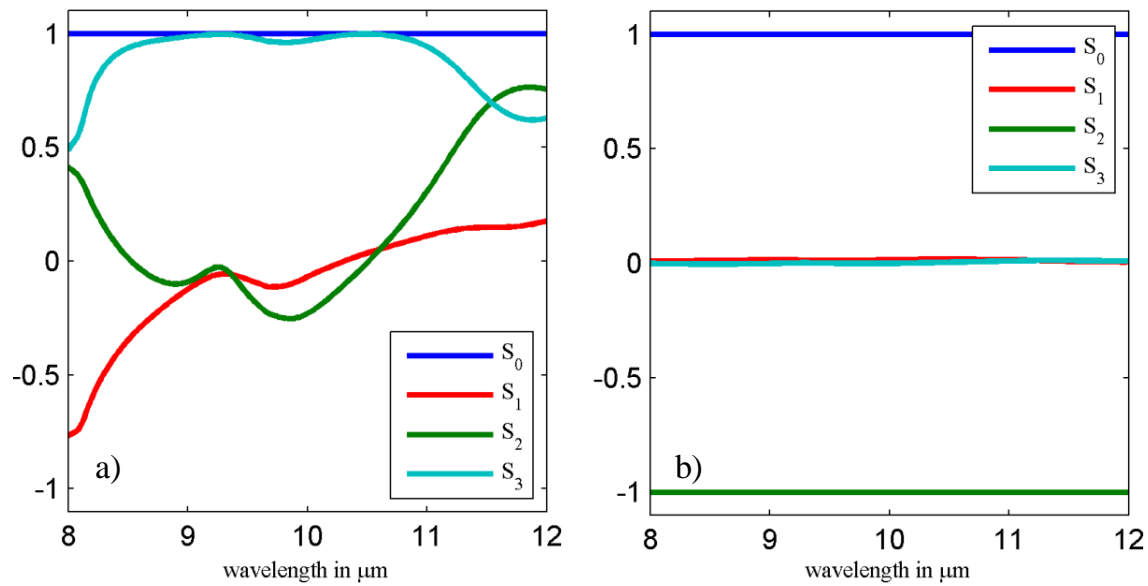

Fig. 2. Modeled Stokes parameters for a) circular spiral, and b) L-shaped wedge elements.

Simulation by HFSS does not account for fluctuational electrodynamics and assumes absolute correlation between orthogonal field components of the thermally emitted radiation, which is contrary to the FDT even for the subwavelength geometries of the proposed designs in Fig. 1. With this assumed coherence, Fig. 2 shows that the spiral elements reveal a high degree of RHCP indicated by the near-unity value of $S_{3}$, whereas the L-shaped wedge elements emit a broad spectral signature of $-45^{\circ}$ linear-polarized radiation $\left(S_{2}=-1\right)$. We will see in the measurements of these structures that the assumptions of the FDT are verified, and this polarization behavior is not observed experimentally.

To design a structure that can emit $\mathrm{CP}$ radiation in spite of the constraints imposed by the FDT, we begin with a wire-grid polarizer in contact with the thermal source. We have previously demonstrated [25] that such a structure emits primarily LP radiation when thermally excited. This LP characteristic does not require any cross-polarized coherence, and thus does not contradict the FDT. As shown in Fig. 3, there is a dielectric standoff layer between this grid and a meanderline layer [38,39], which is designed to give quarter-wave plate (QWP) behavior. The grid and the meanderline layer are oriented at $45^{\circ}$. The meanderline FSS generates CP radiation in transmission by splitting equal components of $45^{\circ}$ LP radiation, and imposing a $90^{\circ}$ phase shift between the two orthogonal field components.

a)

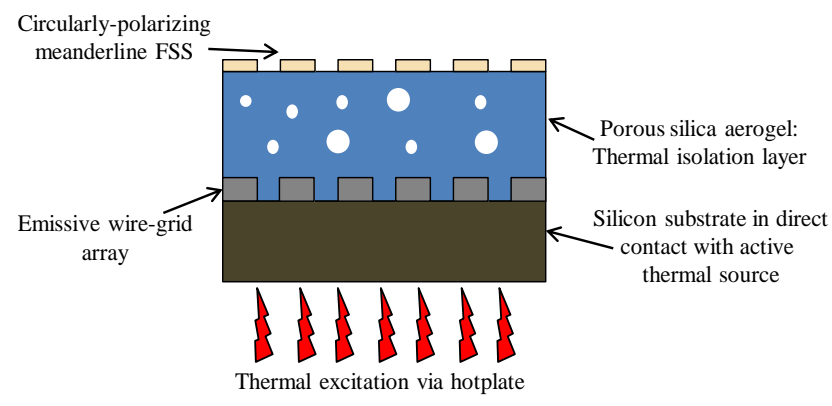

b)

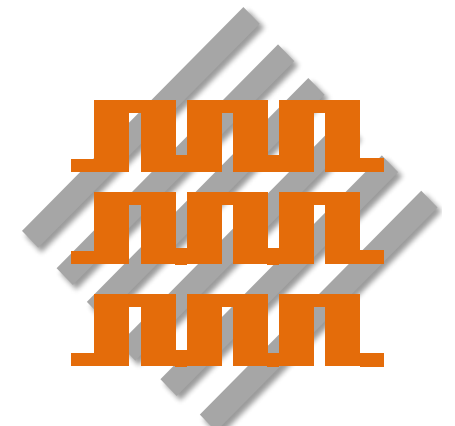

Fig. 3. Depiction of a) cross-sectional profile of multilayer structure for generating CP thermal light. The silicon substrate is heated by an active thermal source (hotplate), which generates a linear-polarized signature upon thermal excitation of the wire-grid array. The wire-grid array (in grey) is rotated by $45^{\circ}$, as shown in b), so that the CP meanderline FSS structure (in orange) is able to generate circular polarization upon transmission of the $45^{\circ}$-tilted linear polarized emission. The aerogel helps to thermally isolate the $\mathrm{CP}$ meanderline layers from the effects of thermal conduction, and subsequently the consequences of the FDT. 
We use a silica aerogel as the dielectric layer. This is a porous material with extremely low thermal conductivity [40-42], which acts as a thermal barrier between the active thermal source and the top layer of metal comprising the meanderline. Recent time domain thermoreflectance (TDTR) analyses of similarly prepared aerogel thin films have reported thermal conductivity values of $0.05-0.35 \mathrm{~W} / \mathrm{m}-\mathrm{K}[43,44]$. For the films used in this study, a thermal conductivity of $\sim 0.053 \mathrm{~W} / \mathrm{m}-\mathrm{K}$ is expected [44], roughly double the conductivity of an air gap, which is sufficient for obtaining a finite thermal gradient over the thickness of the aerogel dielectric layer. Therefore, the primary reason for the inclusion of silica aerogel films is to maintain a steady-state temperature difference between the thermal source and the meanderline while enabling sufficient power transmission through the structure. As shown on Fig. 4, the optical constants of silica aerogel permit ample transmission throughout most of the LWIR band.

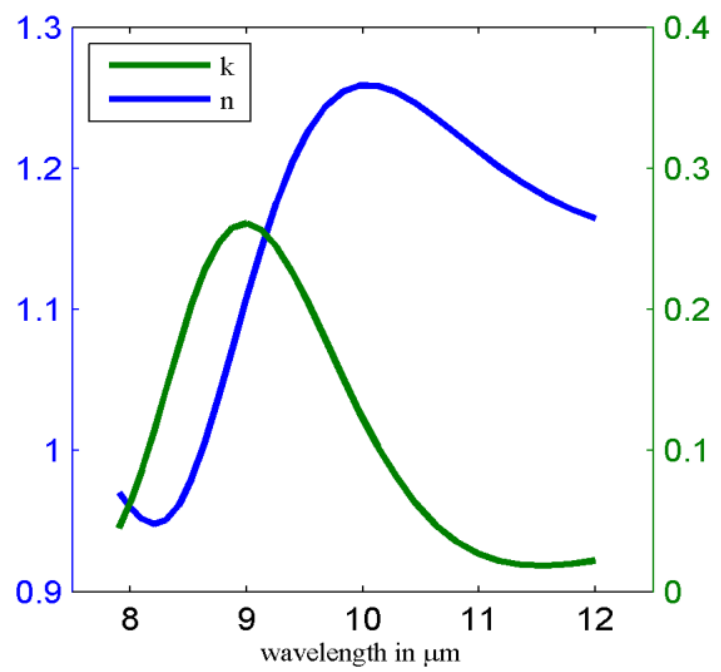

Fig. 4. Optical constants of silica aerogel measured by infrared ellipsometry [45].

We have observed that meanderline structures have a strong LP component of thermal emission, polarized along the long direction of the wires. Thus, measurements will show both $\mathrm{CP}$ and LP emission. To emphasize the CP emission that is transmitted by the meanderline, it is desirable to lower the temperature of the top layer, compared to that of the wire grid.

\section{FSS fabrication}

The FSS elements depicted in Fig. 1 were fabricated on a dielectric standoff layer of BCB above a reflective metallic $\mathrm{Al}$ groundplane. Initially, the $\mathrm{Al}$ groundplane layer was deposited on the polished side of a single-sided-polished (SSP) silicon (Si) wafer. Afterwards, a coating of $\mathrm{BCB}$ totaling $1.2 \mu \mathrm{m}$ in thickness was spun on and cured on top of the Al groundplane. The Ti-metal resonant elements seen in Fig. 5 were written using a Leica EBPG 5000 + e-beam lithography system, and finished with a liftoff process.

\#148230 - \$15.00 USD

(C) 2011 OSA
Received 2 Jun 2011; revised 20 Jun 2011; accepted 21 Jun 2011; published 28 Jun 2011

1 July 2011 / Vol. 1, No. 3 / OPTICAL MATERIALS EXPRESS 473 


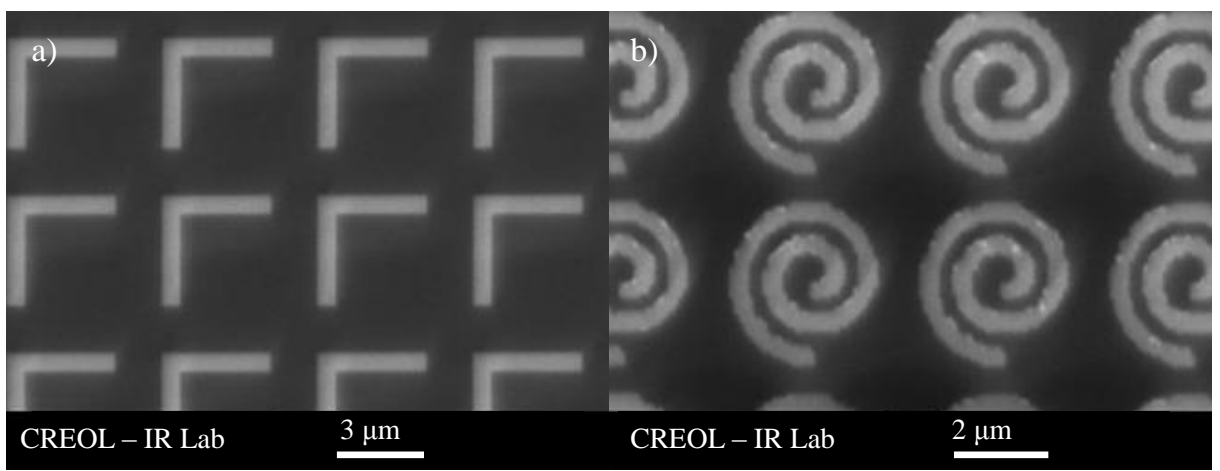

Fig. 5. SEM images of fabricated planar periodic FSS structures corresponding to a) L-shaped wedge elements, and b) circular spiral elements. Dimensions of the elements are given on the corresponding diagrams in Fig. 1.

The structure shown in Fig. 3 was fabricated in a similar fashion. As before, a plasmacleaned SSP Si wafer was utilized for the fabrication of the entire structure, with the linearpolarized emissive wire-grid array elements being directly patterned and metalized with $\mathrm{Al}$ on the polished side of the Si wafer. The wire elements were exposed with a $0.5 \mu \mathrm{m}$ linewidth and an array pitch of $1 \mu \mathrm{m}$. Afterwards, the aerogel layer was deposited on to the patterned wire-grid surface by using a layer-by-layer spin-coating technique. The silica aerogel formula was of quite low viscosity, necessitating multiple 1500-rpm spin-coating steps to deposit the required thickness of aerogel, which was around $1 \mu \mathrm{m}$. Then the stack was baked at $120^{\circ} \mathrm{C}$ for 10 minutes to drive out the ethanol solvent, leaving the porous silica-aerogel film. To cap and planarize the resulting porous surface, a layer of BCB approximately $350 \mathrm{~nm}$ thick was spun on to the aerogel film, and cured. As shown schematically in Fig. 6, the BCB penetrates into the surface of the silica aerogel, but then prohibits any further flow into the aerogel film. This procedure provided a surface suitable for the lithographic steps required to fabricate the meanderline elements on top of the BCB capping layer.

a)

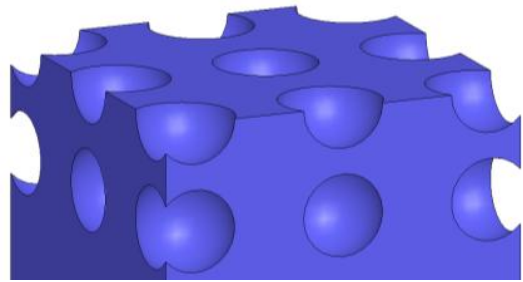

b)

Porous aerogel surface with BCB capping layer

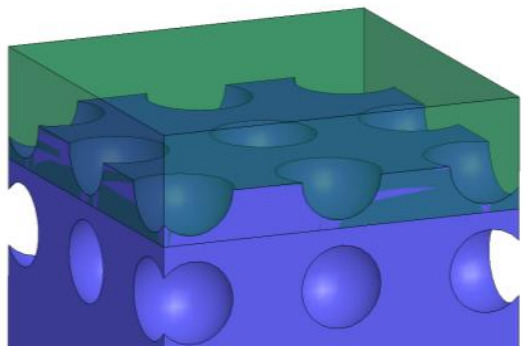

Fig. 6. Schematic of a) porous silica aerogel surface without any capping layer, and b) aerogel surface with a layer of $\mathrm{BCB}$ that effectively caps the aerogel film and planarizes the surface.

The dimensions of the single-layer $\mathrm{Al}$ meanderline structure are given as annotations on the SEM micrograph, Fig. 7. Although two or more meanderline layers could have been used to provide better CP transmission [39], we found it difficult to fabricate more than one layer on top of the aerogel film. This was caused by a tendency toward surface cracking upon deposition of any successive dielectric films or metalized layers. 


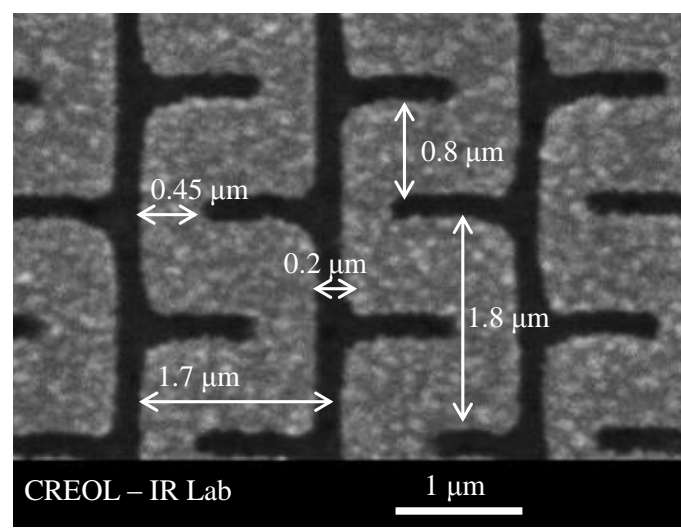

Fig. 7. SEM micrograph of meanderline FSS QWP layer with corresponding array dimensions. The elements were fabricated via e-beam lithography and patterned with metallic Al.

\section{Broadband polarimetric characterization}

The fabricated FSSs were then characterized using broadband imaging polarimetry. This technique serves to filter particular states of polarization through specific combinations and orientations of analyzing polarizers and quarter-wave plate optical components [22-24,26]. The main objective of such methods is to directly image the state of polarization on the focalplane array (FPA) of an 8-12 $\mu \mathrm{m}$ camera (EMX uncooled LWIR imager, model \# 01031), thus analyzing the detected irradiance into components associated with a given state of polarization. The setup for this polarization-based imaging (Fig. 8) is similar to a configuration for measuring the Stokes parameters, with the proviso that the polarization components must be broadband, consistent with the spectral bandwidth of the camera.
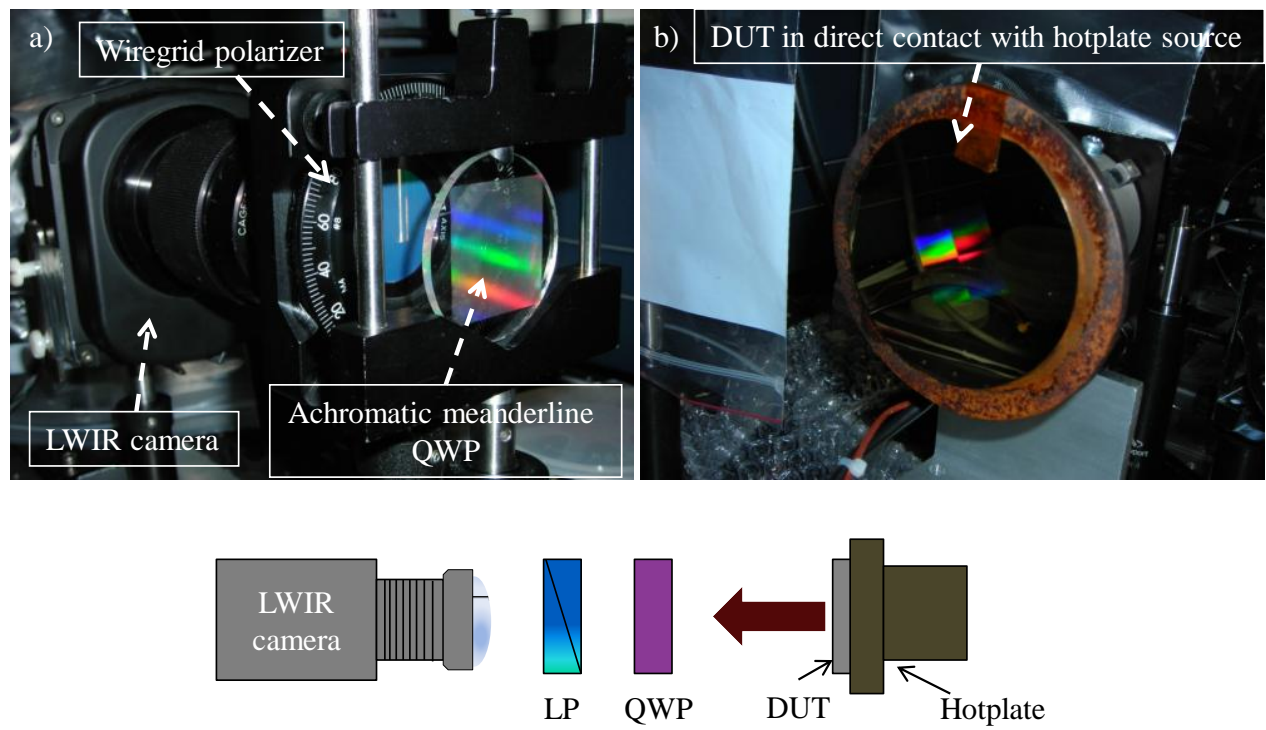

Fig. 8. Broadband imaging polarimetry setup with a) LWIR camera and polarization optics, and b) device under test (DUT) in direct contact with hotplate. The schematic represents the polarimetric system with the wire-grid linear polarizer (LP) and achromatic meanderline quarter-wave plate (QWP) as the analyzing polarization components. 
It should be mentioned that the device under test (DUT) in part b) of Fig. 8 was placed in direct contact with the active hotplate source, thereby achieving the desired configuration of conductive thermal excitation. The hotplate was brought up to a temperature of $45^{\circ} \mathrm{C}$, which was enough to thermally excite the planar periodic FSS arrays and image the emitted fields with the LWIR camera. The heated DUT was imaged through the polarization optics of part a), including the achromatic multilayer meanderline QWP [39], such that the polarizationspecific components were detected by the camera's FPA. Discrete states of polarization were isolated by rotating the wire grid linear polarizer through specific angles, while the meanderline QWP was inserted and removed from the system for switching between $S_{3}$ and $S_{2}$-based polarization imaging, respectively. A series of images was taken for each combination of linear polarizer angle and insertion of the broadband achromatic QWP, with the total combined image information being translated into each component of the Stokes vector:

$$
\begin{gathered}
S_{0}=I\left(\theta=0^{\circ}, \phi=0\right)+I\left(\theta=90^{\circ}, \phi=0\right), \\
S_{1}=I\left(\theta=0^{\circ}, \phi=0\right)-I\left(\theta=90^{\circ}, \phi=0\right), \\
S_{2}=I\left(\theta=45^{\circ}, \phi=0\right)-I\left(\theta=135^{\circ}, \phi=0\right), \\
S_{3}=\left(I\left(\theta=45^{\circ}, \phi=\pi / 2\right)-I\left(\theta=135^{\circ}, \phi=\pi / 2\right)\right) / a^{2} .
\end{gathered}
$$

In the previous formulas, $I$ corresponds to the measured irradiance for a given configuration of linear polarizer and QWP, $\theta$ is the rotation angle of the analyzing linear polarizer, $\varphi=\pi / 2$ is indicative of the insertion of the achromatic QWP that provides a $\pi / 2$ phase shift upon transmission, and $a^{2}$ is the attenuation of the beam as it passes through the meanderline QWP component. The QWP device was inserted into the polarimetric system for imaging of $S_{3}$ only, so an appropriate attenuation constant needed to be introduced to account for the absorption of IR radiation as it passed through the lossy meanderline QWP. These preceding formulas were used to analyze the image information and ultimately produce an area-averaged Stokes vector component of the thermally emitted electromagnetic fields from the subwavelength FSS structures. The following section will detail this intensity-based polarization analysis procedure, along with the results that were acquired from evaluation of the images taken from the broadband polarimetry system.

\section{Image analysis}

The image-analysis methodology employed for measurement of the Stokes vector components is illustrated in Fig. 9. The outlined box with the corresponding title of 'noise' represents the area of the image that was averaged based upon the numerical equivalent of the gray-scale intensity, with 0 being the lowest value, and 255 as the maximum. This baseline noise value was averaged over all successive images corresponding to each irradiance-based measurement of the Stokes parameters given by Eq. (8). There were a total of 6 images taken for a series of Stokes vector coefficients, with each image having its average noise value subtracted from the entire matrix of numerical gray-scale irradiance information.

\#148230 - \$15.00 USD

(C) 2011 OSA
Received 2 Jun 2011; revised 20 Jun 2011; accepted 21 Jun 2011; published 28 Jun 2011

1 July 2011 / Vol. 1, No. 3 / OPTICAL MATERIALS EXPRESS 476 


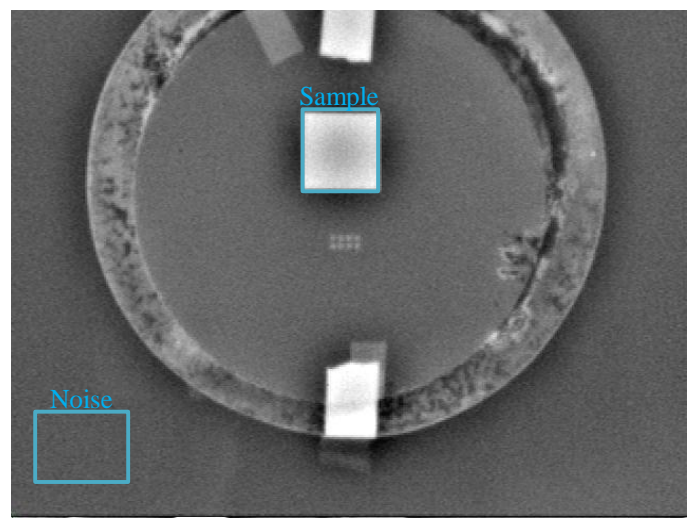

Fig. 9. Example image taken from polarimetric system with annotated boxes outlining the areas that were selected for noise averaging and the sample area.

The second outlined box with the title of 'sample' in Fig. 9 indicates the sample area of the planar periodic FSS structure that was evaluated. Upon subtraction of the baseline noise, it was observed that the resulting gray-scale irradiance, shown in Fig. 10, varied somewhat over the area of the sample. This variation in irradiance directly corresponds to the temperature gradient of the FSS grid area, which exhibits irregularities due to inhomogeneous effects, such as scattering from structural array defects and non-uniform heat transfer. Although such details are apparent in a single image, polarimetric information relies upon the intensity contrast between successive images of orthogonal polarizations. The ensuing contrast between orthogonal states results in normalized Stokes parameters that can be averaged over the total area of the FSS array. Thus, the Stokes vector components were computed with the 2dimensional matrices that correspond to the noise-subtracted gray-scale irradiance from the entire area of the FSS sample. After using the formulas given in Eq. (8), the arrays of Stokes coefficients were then averaged to yield a single value that represents the area-averaged polarization-based irradiance signal received by the LWIR thermal camera.
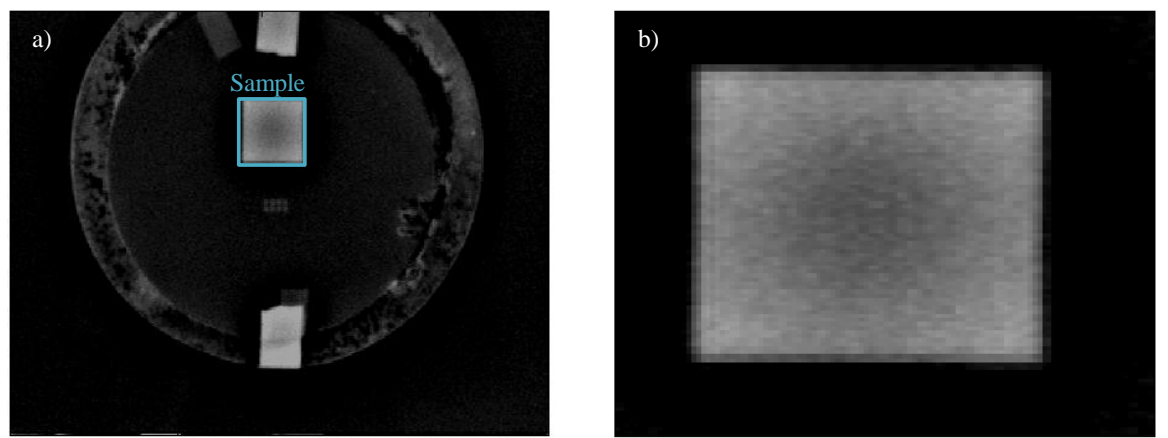

Fig. 10. Same image as Fig. 9, but with the baseline noise subtracted from the data. Part a) shows the sample area as before, with part b) as a zoomed-in image of the planar FSS area. Note the variation in local intensity of the thermally emitted fields over the area of the FSS.

Upon evaluation of the percent transmission through the achromatic meanderline QWP [39], which yields the attenuation constant $a^{2}$, the normalized Stokes vector was obtained by inserting the appropriate gray-scale intensity matrices into Eq. (8) and dividing each areaaveraged coefficient by $S_{0}$ [26]. This procedure was followed for each FSS device measured.

We will present our measured results in terms of the total degree of polarization (DOP), total degree of unpolarized radiation (DOUP), the total degree of linear polarization (DOLP), 
and the total degree of circular polarization (DOCP). In terms of the Stokes parameters of Eq. (8), these quantities are defined as [26]:

$$
\begin{gathered}
D O P=\sqrt{S_{1}^{2}+S_{2}^{2}+S_{3}^{2}} / S_{0}, \\
D O C P=\left|S_{3} / S_{0}\right|, \\
D O L P=\sqrt{D O P^{2}-D O C P^{2}}=\sqrt{S_{1}^{2}+S_{2}^{2}} / S_{0}, \\
D O U P=\sqrt{1-D O P^{2}} .
\end{gathered}
$$

Alternatively, the DOCP given by Eq. (9b) can be completely described in terms of images taken with the analyzing polarizer at the $45^{\circ}$ and $135^{\circ}$ angles, and with the achromatic multilayered meanderline QWP inserted into the system. In this way, the attenuation coefficient $a^{2}$ does not need to be taken into account, since the total normalized DOCP is evaluated with respect to the total intensity that passes through the linear polarizer and meanderline QWP components

$$
D O C P=\left|\frac{\left(I\left(\theta=45^{\circ}, \phi=\pi / 2\right)-I\left(\theta=135^{\circ}, \phi=\pi / 2\right)\right)}{\left(I\left(\theta=45^{\circ}, \phi=\pi / 2\right)+I\left(\theta=135^{\circ}, \phi=\pi / 2\right)\right)}\right| .
$$

This alternate expression for the DOCP was used in our computation of results.

\section{Measurement results and discussion}

The normalized area-averaged Stokes vector coefficient results are shown in Table 1, for the circular-spiral and L-shaped-element FSS arrays as well as the multilayer CP FSS structure.

Table 1. Measured Polarimetric Data for the Structures Investigated*

\begin{tabular}{cccc}
\hline Polarimetric data & $\begin{array}{c}\text { L-shaped } \\
\text { elements }\end{array}$ & Spiral elements & $\begin{array}{c}\text { Multilayer FSS } \\
\text { structure }\end{array}$ \\
\hline$S_{1} / S_{0}$ & $<0.05$ & -0.19 & 0.63 \\
$S_{2} / S_{0}$ & 0.07 & -0.13 & -0.44 \\
$S_{3} / S_{0}$ & $<0.05$ & $<0.05$ & -0.28 \\
DOCP & $<0.05$ & $<0.05$ & 0.28 \\
DOLP & 0.07 & 0.23 & 0.77 \\
DOP & 0.08 & 0.23 & 0.84 \\
DOUP & 0.99 & 0.97 & 0.55 \\
\hline
\end{tabular}

*average deviation of measured quantities $\approx 0.05$, or $5 \%$

The purpose of the planar FSS designs was to verify the validity of the FDT, such that crosspolarized thermal emission would ultimately be suppressed. The data shown on Table 1 clearly illustrates the lack of correlation between orthogonal surface currents, since the Lshaped wedge element array results in mostly unpolarized radiation, and the circular spiral elements emit negligible CP radiation. Thus, we have experimentally verified that the orthogonal field components are uncorrelated, and that thermal emission of $\mathrm{CP}$ radiation is not feasible with a planar structure. The data for the multilayer CP FSS structure incorporating the 
aerogel isolation layer shows a strong LP emission, which is expected from thermal excitation of the meanderline wires. However, the DOCP for this structure is also significant, at $28 \%$. To our knowledge, this is the first time that a thermally emitted CP signature was obtained over a broad spectrum using planar multilayer FSS structures.

\section{Conclusion}

In this paper, we have studied the polarized thermal emission properties of planar periodic FSS structures, specifically in their ability to emit CP radiation that can be detected by broadband imaging polarimetry. We found that, consistent with the FDT, purely planar structures in thermal contact with an elevated-temperature source did not exhibit any significant correlation between cross-polarized components, rendering these structures unable to emit broadband CP radiation. However, we also found that a combination of an LPemitting wire grid and a transmissive meanderline QWP layer for converting LP to CP yielded a $28 \%$ DOCP. The performance of the measured device for radiation of broadband CP depended on an aerogel thermal isolation layer, which allowed a steady-state temperature difference between the LP-radiating grid, and the LP-radiating meanderline layers. Further improvement of the DOCP may be possible with refinement of the silica aerogel process. If thicker layers could be deposited without surface fracturing, then a larger steady-state temperature difference could be maintained, with consequently higher DOCP and lower DOLP.

\section{Acknowledgments}

This research was supported by the Laboratory Directed Research and Development program at Sandia National Laboratories.

Sandia is a multiprogram laboratory operated by Sandia Corporation, a Lockheed Martin Company, for the United States Department of Energy's National Nuclear Security Administration under Contract DE-AC04-94AL85000.

$\# 148230$ - \$15.00 USD

(C) 2011 OSA
Received 2 Jun 2011; revised 20 Jun 2011; accepted 21 Jun 2011; published 28 Jun 2011 1 July 2011 / Vol. 1, No. 3 / OPTICAL MATERIALS EXPRESS 479 